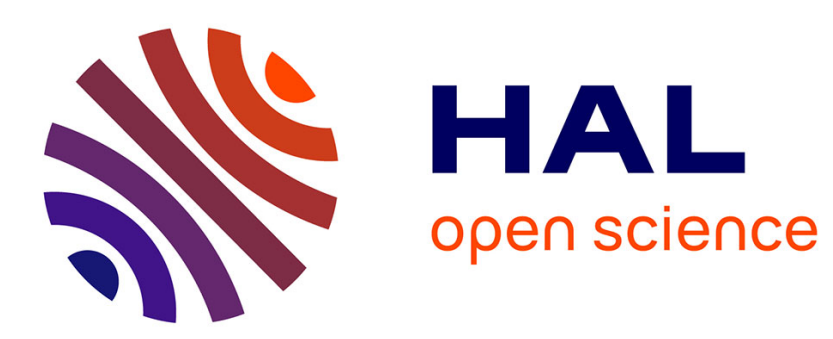

\title{
A semi-autonomous framework for human-aware and user intention driven wheelchair mobility assistance
}

Vishnu Karakkat Narayanan, Anne Spalanzani, Marie Babel

\section{To cite this version:}

Vishnu Karakkat Narayanan, Anne Spalanzani, Marie Babel. A semi-autonomous framework for human-aware and user intention driven wheelchair mobility assistance. IEEE/RSJ Int. Conf. on Intelligent Robots and Systems, IROS'16, Oct 2016, Daejeon, South Korea. pp.4700-4707. hal01355481

\section{HAL Id: hal-01355481 \\ https://hal.inria.fr/hal-01355481}

Submitted on 23 Aug 2016

HAL is a multi-disciplinary open access archive for the deposit and dissemination of scientific research documents, whether they are published or not. The documents may come from teaching and research institutions in France or abroad, or from public or private research centers.
L'archive ouverte pluridisciplinaire HAL, est destinée au dépôt et à la diffusion de documents scientifiques de niveau recherche, publiés ou non, émanant des établissements d'enseignement et de recherche français ou étrangers, des laboratoires publics ou privés. 


\title{
A semi-autonomous framework for human-aware and user intention driven wheelchair mobility assistance
}

\author{
Vishnu K.Narayanan ${ }^{1}$, Anne Spalanzani ${ }^{2}$ and Marie Babel ${ }^{3}$
}

\begin{abstract}
An important aspect to be taken care of while designing assistive robots for mobility is that they need to operate among humans. Thus understanding human spatial social conventions and incorporating them in the assistive solutions, is important. In this paper, we introduce a semi-autonomous framework for assistive wheelchair navigation in human environments, which is driven by the intention of the wheelchair user. Safe and socially compliant motion provided by a user intention driven local motion planner is fused with user teleoperation in order to create such a system. Taking into account the fact that the user is the primary controller, our proposed system aims to provide progressive assistance whenever the user is in danger of collision or at risk of disturbance to other humans. We also thus propose generalized formulations for estimating user intentions and for sharing control within the context of wheelchair mobility assistance, that is adaptable in order to be deployed in real world systems. We then evaluate the proposed framework in simulation in order to obtain a quantitative analysis. We also provide experimental evidence using an off-the-shelf robotized wheelchair equipped with a single 2D laser scanner.
\end{abstract}

\section{INTRODUCTION}

As per the US Census Bureau statistics of 2010, wheelchairs provide assistance to 3.6 million people above the age of 15 [1]. Even though they remain the primary assistive device for mobility, a large number of users, particularly people suffering from motor and/or visual impairments, are unable to utilize wheelchairs in a rather safe and socially compliant manner. Evidently, the advent of powered wheelchairs since the late 1970s coupled with recent advances in robotics has led to research on Intelligent Wheelchairs [2]-[11]. A variety of initiatives like the TAO Project [2], the NavChair [3], European FP7 Radhar project [4] and the SYSIASS project [5] were indeed able to design wheelchair systems that take partial/full control from the user for safe mobility assistance.

A key issue that hinders the adoption of such technologies in the real world is that they need to operate in mostly human crowds. But proxemic issues are not usually explicitly accounted for in the design. Traditional robotic objectives such as taking the shortest path toward a goal are no longer optimal in such cases [8]-[10], [12].

In addition, since it is also well known that individual mobility is an integral part of the mental and social well-being of a disabled person [13], it is also better to assign primary control over the wheelchair motion to the user [7].

\footnotetext{
${ }^{1}$ Vishnu K.Narayanan is with Inria Rennes - Bretagne Atlantique, France. vishnu.karakkat-narayanan at inria.fr

2 Anne Spalanzani is with Univ. Grenoble Alpes and Inria Grenoble Rhone Alpes, France

${ }^{3}$ Marie Babel is with INSA Rennes and Inria Rennes - Bretagne Atlantique, France
}

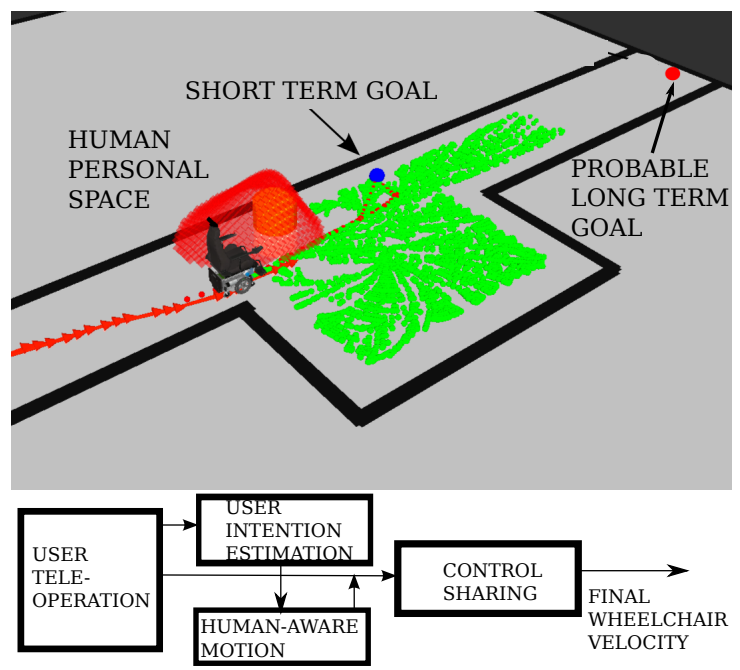

Fig. 1. Robots in human environments need to respect the virtual socia spaces that is created when humans are present. The Figure shows a robotic wheelchair following a corridor by planning a trajectory (in red) toward a short term goal (in blue) while taking into account these spatial social constraints, within a 2D configuration space. We augment the user teleoperation with velocities coming from the motion planner while regularly predicting these short term goals that drive the said motion planner.

We thus introduce a semi-autonomous framework for wheelchair mobility assistance that addresses the present shortcomings and that is adaptable in order to be deployed in real world systems. This is addressed by integrating three key capabilities viz. human-awareness, short term user intention estimation and a generalised control sharing formalism into wheelchair navigation (see Fig. 1). We can design humanaware motion by constraining a traditional motion planning algorithm with the idea of virtual social spaces that is created in human presence [14]. This reduces the risk of disturbance to other humans (henceforth termed risk of disturbance) [8].

Estimating user intention, on the other hand, can be quite challenging [6], [8], [9], [11]. Since the objective is to assign primary control to the user, predicting short term navigation goals is sufficient. Short term goals predicted at regular intervals tend to be sub-optimal goals that are agnostic but inherently compatible to the user's long term goal. Such goals, which depend on the wheelchair state and the direction of user intended motion at the time of prediction, can serve as destinations for the constrained motion planner. User teleoperation velocity can then be augmented with the velocity coming from the goal driven constrained motion planner so that progressive assistance can be provided in order to reduce risk of collision and to reduce risk of disturbance to other humans. 
Also, an essential issue to consider while designing mobility assistance systems is to facilitate the learning of user habits [15], [16]. We also provide a case for learning user habits (i.e. learning frequent goals as well as learning when and how to assist) alongside the proposed framework. Before moving onto the design, we present the related research within the relevant domains and define our contributions.

\section{RELATED WORK AND CONTRIBUTIONS}

\section{A. Human-aware Motion Planning}

Majority of the literature on human-aware path/motion planning is derived from the theory of proxemics as put forth by anthropologist P. E. Hall [14]. Social robots that navigate in human environments need to respect the virtual social spaces around humans in order to reduce discomfort and produce a human-aware motion [8], [9], [17], [18]. Surveys [19], [20] of human-aware robot navigation identified three key objectives viz. Comfort, Naturalness and Sociability that drive research in this area. Importantly, using traditional robotic objectives like the shortest path may not be optimal when emphasising human-awareness [10], [12]. Thus, in many previous works in this area, the path planner is designed to generate a humanlike motion either using a specific performance criteria [17], [18], [21], [22] or by using machine learning [12].

Whereas another direction of research focuses on addressing the virtual social spaces in order to reduce discomfort and increase acceptability [8]-[10], [20]. Then in our case, it is advantageous to incorporate this idea of virtual social spaces as the user is deemed have primary control over the wheelchair. We therefore utilize a risk-based RRT (Rapidly Exploring Random Trees) motion planner within which virtual social spaces are explicitly considered [8]. The planner generates safe velocities toward a pre-set goal while ensuring that the trajectory taken by the wheelchair is socially compliant (i.e. does not induce discomfort to other humans).

\section{B. User Intention Estimation}

Moving onto the issue of user intention consideration, initial designs for wheelchair navigation assistance provided higher level control to the user where the final goal was given by the user and the wheelchair performed as an autonomous robot [2], [3], [9]. Following this, untraditional interfaces such as Brain-Controlled Interfaces (BCIs) [11] and RGB-D sensors able to track faces [9] were utilized to infer user intention in the form of topological poses in the wheelchair configuration space. Approaches that relied on plan/action recognition [7], [23] (eg. providing assistance for a specific task such as doorway traversal) also were introduced. Finally learning techniques such as using Partially Observable Markov Decision Processes [15], or Leaning from Demonstration [16] were investigated for long term intention prediction.

All intention prediction mechanisms described above are highly deterministic and do not have the capability of estimating user intention uncertainty which may cause much discomfort to the user if the wheelchair does not behave as intended. A Bayesian based approach was presented in [24] where the uncertainty in user intention was explicitly modelled. This allowed for a more user centric design. We here argue that the user intention uncertainty can be avoided by only predicting short term goals that are sub-optimal and agnostic to the user's long term intention. We use Bayesian reasoning to infer such short term goals where they only depend on the current wheelchair state and the current direction of the user's intended motion. The proposed formulation can be used to predict goals at regular intervals and also be used to learn the user's frequent goals, which in turn may improve performance.

\section{Control Sharing}

As stated earlier, intelligent wheelchairs initially were designed as autonomous robots where the user was given the freedom to choose his final destination [2], [3], [9]. Consequently, lower level control in the form of augmenting the user teleoperation, was introduced [4], [6], [7], [11], [16], [23]. The final wheelchair velocity was calculated using a linear control sharing formalism of the general form $(1-\alpha) \mathbf{v}_{o p}+\alpha \mathbf{v}_{r}$ with $\mathbf{v}_{o p}$ and $\mathbf{v}_{r}$ representing the user teleoperation and the corrective velocity respectively (eg. velocity coming from a motion planner). Here $\alpha$ henceforth termed the assistance factor represents the amount of control the autonomous controller has at a particular instant as it translates to an allocation weight for each contributing velocity.

This formalism has been researched thoroughly in the field and in majority of the studies [4], [6], [16], [23], the assistance factor is set as a scalar (i.e all the Degrees of Freedom (DOFs) of the wheelchair respond to the same assistance factor) and is determined according to the specific needs of the control system. We extend this formalism towards a more general, stable and user centric design. Our previous work [7] introduced a concept for determining the factor as a function that progressively moves from Null to Identity (i.e. from full control to the user to full control to the system) when there is danger of collision. A progressive increase (or decrease) in control sharing is key for better quality of driving. This idea was verified within a vision-based system for assistance in corridor following. In the present case, we propose an adaptive formulation for control sharing depending on the risk of collision and the risk of disturbance to other humans.

\section{PRELIMINARIES}

Thus, in Section IV, we introduce the general ideas of human social spaces as well as present an overview of the socially constrained local motion planner proposed in [8]. Section $\mathrm{V}$ then formalizes the user intention estimation process that generates short term goals which in turn drives the motion planner. We conclude the section by proposing a general shared control strategy that can fuse user teleoperation with velocities generated from the motion planner in a progressive manner, while preserving primary control with the user.

Consequently, we model the wheelchair as a nonholonomic unicycle-type robot moving on a horizontal or inclined plane, while assuming that the user teleoperates it via a traditional 2D joystick. We thus have the following definitions: 
- $\mathbf{v}=[v, \omega]^{T}$ : The wheelchair control velocity with its translational $(v)$ and angular $(\omega)$ components.

- $\mathbf{v}_{o p}=\left[v_{o p}, \omega_{o p}\right]^{T}$ : The user teleoperation velocity coming from a traditional 2D joystick.

- $\mathbf{v}_{r}=\left[v_{r}, \omega_{r}\right]^{T}$ : The velocity from the constrained local motion planner.

If the wheelchair navigates within a $2 \mathrm{D}$ configuration space ( $\mathbf{S E}(2)$ represented by $(x, y, \phi))$ we have,

- $\mathbf{X}_{t}=\left(x_{t}, y_{t}, \phi_{t}\right)$ : The wheelchair configuration at a particular instant $t$.

- $\mathbf{X}_{g}=\left(x_{g}, y_{g}, \phi_{g}\right)$ : The estimated short term goal towards which the user intends to navigate.

We assert that at any instant $t$ of goal prediction, $\mathbf{X}_{g}$ is a function of $\mathbf{X}_{t}$ and $\phi_{t}^{*}$ where $\phi_{t}^{*}$ is the angle between the $2 \mathrm{D}$ joystick direction at $t$ with respect to the $x$ axis of the space (see Fig 4). Our goal is then to fuse $\mathbf{v}_{o p}$ and $\mathbf{v}_{r}$ in order to derive a progressive assistive system for semi-autonomous navigation among human crowds while estimating $\mathbf{X}_{g}$ at regular intervals.

\section{Planning Under Social Constraints}

\section{A. The idea of social spaces}

Since intelligent wheelchairs need to operate in populated environments, it is essential to encode compliant social behaviours within the control algorithms. For example, people tend to become uncomfortable if they are approached at a distance that is deemed too close. The level of discomfort generally depends on the personal space of an individual or on the interaction space of a group of humans [14]. The notion of personal and interaction spaces can be explicitly characterized using general models.

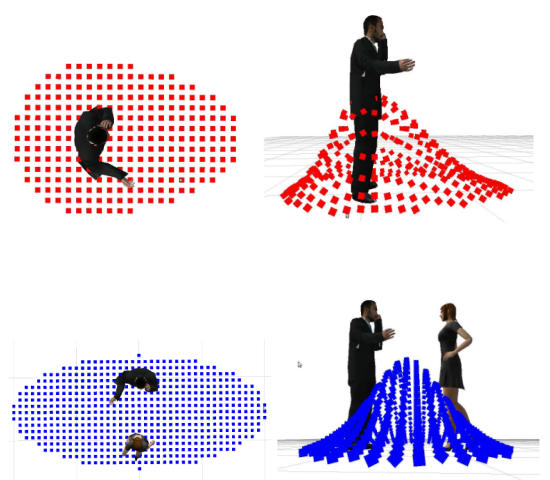

Fig. 2. The Personal Space (top) of an individual and the Interaction Space (bottom) of a group can be modelled within a 2D configuration space as shown above. The height of the points in the figures represents the relative risk of disturbance to the humans at that position. For a single human, maximum disturbance is located at human position. For a group, maximum disturbance is located at the group center.

Assuming that a robot is operating in a $2 \mathrm{D}$ configuration space, the models of personal and interaction spaces are given in Fig. 2. The height of the points represent the relative risk of disturbance to the person/s (in terms of comfort). The personal space can be modelled as a blending of two
Gaussian functions, one for the front of the human and one for the rear (see Fig. 2 top). Evidently, the Gaussian for the front is wider than the one for the rear. On the other hand, the interaction space is represented by a two dimensional Gaussian (see Fig. 2 bottom). The Gaussian is centered at the group focus of attention which is the group point of visual and cognitive attention. This point in turn can be extracted from the orientation of the humans within the group. These models of social spaces can be directly transformed to cost functions that allows a path planner to plan optimal trajectories in order to reduce the risk of disturbance [8].

\section{B. Constraining the motion planner}

RiskRRT [25] is a local path planner that explores the environment using a Rapidly Exploring Random Tree that is constantly updated with perceived data as well as obstacle motion predictions. It is an extension of the popular RRT algorithm where the likelihood of an obstacle's future trajectory and the risk of collision are taken into account. The tree (comprising of branches corresponding to a set of topological poses in the 2D configuration space) is grown in a random fashion but is heavily biased towards a goal, that is checked for at each iteration. The best trajectory or path in the tree is the one with the least farthest from the goal (in terms of Euclidean distance) and which carries the lowest risk of collision.

The risk of disturbance is then integrated for each person and each interaction as the probability of collision with virtual dynamic objects. In such a case the models of the social spaces serve as cost functions for the probability of occupancy. Fig. 3 shows the execution of the path planner within a specific scenario. Further details regarding the algorithm design as well as a primer on how to detect and represent social spaces are provided in [8]. Finally, in order to traverse the path generated by RiskRRT, kinematically permissible velocities $\left(\mathbf{v}_{r}\right)$ are generated using a well defined stable tracking algorithm proposed by Kanayama et.al. [26].

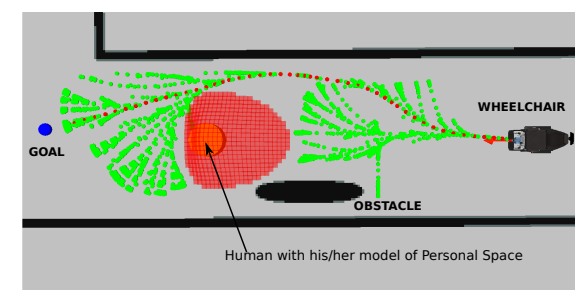

Fig. 3. The risk-based RRT planner grows trees heavily biased toward a preset goal taking into account the perceived information. The best trajectory (branch) of the tree (in red) among the possible trajectories (in green) is the one which is closest to the goal and which carries the lowest risk of collision and the lowest risk of disturbance to other humans.

\section{SEmi-Autonomous FRAMEWORK}

The semi-autonomous framework comprises of two components: The user intention estimation process that generates short term goals which drives the motion planner briefed earlier and the shared control system that fuses the velocities generated from the motion planner in order to augment the user teleoperation. 


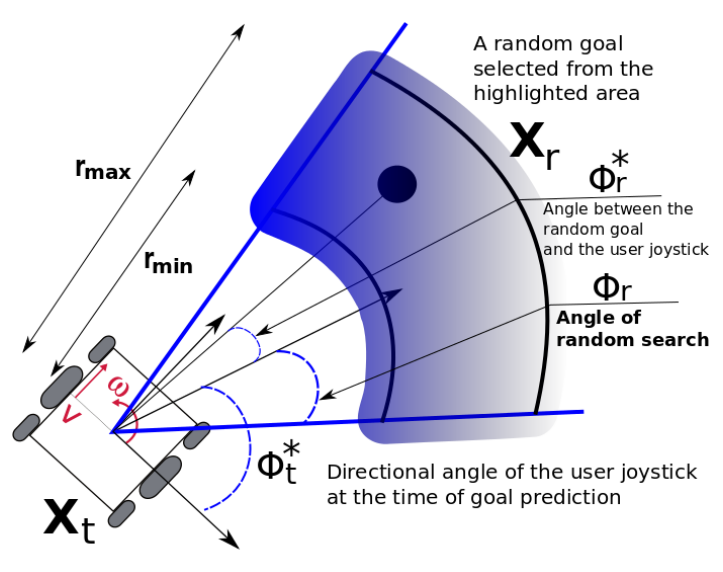

Fig. 4. Random short term goals: A random 2D point $\left(x_{r}, y_{r}\right)$ is searched from within the circular segment as shown in the figure and the random goal $\mathbf{X}_{r}$ is set as $\mathbf{X}_{r}=\left(x_{r}, y_{r}, \phi_{t}^{*}\right)$. The distances $r_{\min }$ and $r_{\max }$ as well as the search angle $\phi_{r}$ determine the size of the search area. The angle between the random goal and the user joystick direction $\phi_{r}^{*}$ is also shown here (see Eqn. (4)).

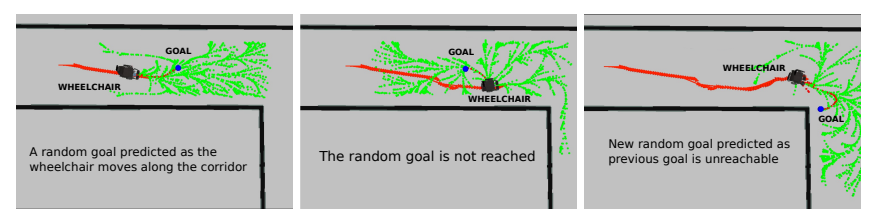

Fig. 5. Random short term goal predictions: A sequence depicting a wheelchair moving within a corridor and into a doorway

\section{A. User Intention Estimation}

We estimate the user intention as topological poses within the configuration space which serve as short-term goals for the motion planner that is in turn used to augment the user teleoperation. We here propose a formulation for predicting goals that nullifies user intention uncertainty i.e. short term goals that are agnostic but compatible with the user's long term intention. In order to generalize, we can consider two scenarios:

1. The wheelchair operates in a constrained and mapped environment with a list of frequent user destinations previously learned or determined.

2. The wheelchair operates in an unconstrained and unmapped environment.

Assuming $\mathbf{X}_{r}=$ function $\left(\mathbf{X}_{t}, \phi_{t}^{*}\right)$ a random goal estimated at each instance of goal prediction (see Fig 4) and $\left\{\mathbf{X}_{1}, \mathbf{X}_{2} \ldots . \mathbf{X}_{n}\right\}$ be the set of $n$ determined/learned goals, then we can define a set $\mathbf{X}_{\mathbf{G}}=\left\{\mathbf{X}_{r}, \mathbf{X}_{1}, \mathbf{X}_{2} \ldots . . \mathbf{X}_{n}\right\}$ of $n+1$ goals. The short term goal $\mathbf{X}_{g}$ can then be formulated as

$$
\mathbf{X}_{g}=\underset{\mathbf{X}_{i} \in \mathbf{X}_{\mathbf{G}}}{\arg \max } P\left(\mathbf{X}_{i} \mid \mathbf{X}_{t}, \phi_{t}^{*}\right)
$$

Thus, $\mathbf{X}_{g}$ is the goal that maximises the posterior probability $P$ of selecting a goal $\mathbf{X}_{i}$ given the current wheelchair configuration $\mathbf{X}_{t}$ and the user joystick directional angle $\phi_{t}^{*}$ at the prediction instant $t$. In order to determine $P\left(\mathbf{X}_{i} \mid \mathbf{X}_{t}, \phi_{t}^{*}\right) \forall i$, we can use Bayes' theorem:

$$
P\left(\mathbf{X}_{i} \mid \mathbf{X}_{t}, \phi_{t}^{*}\right)=\frac{P\left(\phi_{t}^{*} \mid \mathbf{X}_{t}, \mathbf{X}_{i}\right) P\left(\mathbf{X}_{i} \mid \mathbf{X}_{t}\right)}{P\left(\phi_{t}^{*} \mid \mathbf{X}_{t}\right)} .
$$

The above relation can be normalized using a constant $\eta$ as

$$
P\left(\mathbf{X}_{i} \mid \mathbf{X}_{t}, \phi_{t}^{*}\right)=\eta P\left(\phi_{t}^{*} \mid \mathbf{X}_{t}, \mathbf{X}_{i}\right) P\left(\mathbf{X}_{i} \mid \mathbf{X}_{t}\right) .
$$

$P\left(\phi_{t}^{*} \mid \mathbf{X}_{t}, \mathbf{X}_{i}\right)$ represents the probability of the user assigning a joystick directional angle $\phi_{t}^{*}$ given the current configuration $\mathbf{X}_{t}$ and the intended goal $\mathbf{X}_{i}$. Since the prediction window is short term, we design this probability as,

$$
P\left(\phi_{t}^{*} \mid \mathbf{X}_{t}, \mathbf{X}_{i}\right)=\frac{\left(1-\left|\phi_{i}^{*}\right|\right) / \pi}{\sum_{i}\left(1-\left|\phi_{i}^{*}\right|\right) / \pi},
$$

where $\phi_{i}^{*}$ is the angle between the user joystick direction and the goal $\mathbf{X}_{i}$ (see Fig. 4 for a representation of $\phi_{r}^{*}$ where $i=r$ ). Thus at each instant we predict the short term goal $\mathbf{X}_{g}$ as,

$$
\mathbf{X}_{g}=\underset{\mathbf{X}_{i} \in \mathbf{X}_{\mathbf{G}}}{\arg \max } \frac{\left(1-\left|\phi_{i}^{*}\right|\right) / \pi}{\sum_{i}\left(1-\left|\phi_{i}^{*}\right|\right) / \pi} P\left(\mathbf{X}_{i} \mid \mathbf{X}_{t}\right) .
$$

Discussion: In a constrained and mapped environment where a set of goals are previously learnt or determined, we can postulate that the probability $P\left(\mathbf{X}_{i} \mid \mathbf{X}_{t}\right)$ be assigned using specific criteria with the Euclidean distance norm $(d)$ being the most straightforward where

$$
P\left(\mathbf{X}_{i} \mid \mathbf{X}_{t}\right) \propto \frac{1}{d\left(\mathbf{X}_{t}, \mathbf{X}_{i}\right)}
$$

For any random goal $\mathbf{X}_{r}, P\left(\mathbf{X}_{r} \mid \mathbf{X}_{t}\right)$ can be set as a minimum positive threshold $\delta$ in order to give priority to the learnt or determined goals.

Whereas in unconstrained and unmapped environments, it is evident that the random goals will take priority as there are no pre-set goals to reason over. But estimation of intentions over a long time could provide data that captures user sequential decisions, which can be used to learn user habits. In such a case and since we have a low dimensional state space, frequent goals and the prior $P\left(\mathbf{X}_{i} \mid \mathbf{X}_{t}\right) \forall i$ can be learnt using algorithms for Inverse Reinforcement Learning, as we can effectively argue that the user performs an optimal sequence of actions in order to reach his/her short term goals [27].

In terms of prediction interval, we propose that a new goal be determined if the present goal is reached (i.e. $d\left(\mathbf{X}_{t}, \mathbf{X}_{g}\right)<$ $d_{\text {min }}$ ) or if the distance to the present goal is high enough to be unreachable (i.e $\left.d\left(\mathbf{X}_{t}, \mathbf{X}_{g}\right) \geq d_{\max }\right)$. Here $d_{\min }$ and $d_{\max }$ represent the distances within which the short term estimated goal is to be considered.

Fig. 5 shows some instances of random short term goal predictions for a wheelchair moving within a corridor and into a doorway. Since the random goal is dependent on $\phi_{t}^{*}$, the short term user intention is always respected albeit suboptimal. In addition, since the user is deemed to have primary control of the wheelchair, in many cases the estimated goals (especially if they are random) will not be reached. This is not an issue as a new goal will be predicted as soon as $d\left(\mathbf{X}_{t}, \mathbf{X}_{g}\right)=d_{\max }$.

Therefore user intention uncertainty can effectively be ignored. Moreover, a random goal may also lie within the social 


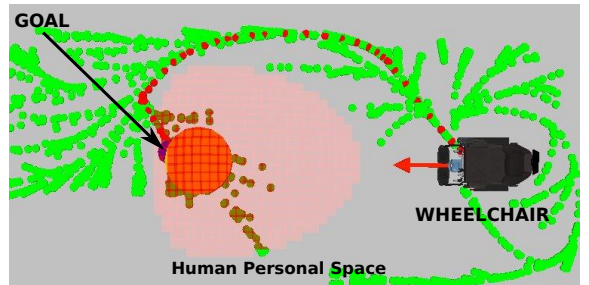

Fig. 6. Here we see a goal being estimated that is in collision with a human. But the motion planner RiskRRT plans a safe and socially compliant path towards the goal. The design of the shared control formalism (see Sec. VB) also ensures that the user teleoperation is augmented in order to evade this danger.

space of a human nearby or on an obstacle (see Fig. 6). This again is not an issue since the motion planner plans safe paths toward the goal and the shared control formalism would ensure that the wheelchair avoids the danger as can be seen from the next subsection.

\section{B. Control Sharing}

The shared control system should take part of the control from the user only in order to reduce the risk of collision or the risk of disturbance to other humans. Also, control sharing should be progressive and smooth in order to ensure that the user is able to perceive the danger and is not frustrated by sudden changes in motion.

Let $\mathbf{A}=\operatorname{diag}\left(\alpha_{v}, \alpha_{\omega}\right)$ be a positive definite diagonal assistance function such that $\alpha_{v} \in[0,1]$ and $\alpha_{\omega} \in[0,1]$, we can design the wheelchair velocity controller as

$$
\mathbf{v}=\left(\mathbb{I}_{2}-\mathbf{A}\right) \mathbf{v}_{o p}+\mathbf{A} \mathbf{v}_{r}
$$

where $\mathbb{I}_{2}$ is a size $2 \times 2$ Identity matrix.

We assert that the only criterion for providing assistance would be the distance to danger. Assume that the closest topological pose where either an obstacle is detected or is part of the human personal or interaction space, is located at a Euclidean distance $d_{\mathbf{A}}$ from the wheelchair (see Fig 7). Then if $\left[d_{\mathbf{A}_{\text {max }}}, d_{\mathbf{A}_{\text {min }}}\right]$ with $d_{\mathbf{A}_{\text {max }}}>d_{\mathbf{A}_{\text {min }}}$ is a pre-defined interval with $d_{\mathbf{A}_{\max }}$ representing the maximum distance from the danger beyond which no assistance should be provided and $d_{\mathbf{A}_{\text {min }}}$ representing the minimum distance from the danger below which the motion controller should essentially take full control, we can assign

$\alpha_{\omega}=\left\{\begin{array}{cl}0 & \text { if } d_{\mathbf{A}}>d_{\mathbf{A}_{\text {max }}} \\ 1+\cos \left(\frac{\pi}{2} \frac{d_{\mathbf{A}}-d_{\mathbf{A}_{\text {min }}}}{d_{\mathbf{A}_{\text {max }}-d_{\mathbf{A}_{\text {min }}}}}\right) & \text { if } d_{\mathbf{A}_{\text {min }}} \leq d_{\mathbf{A}} \leq d_{\mathbf{A}_{\text {max }}} \\ 1 & \text { otherwise }\end{array}\right.$

$$
\alpha_{v}=\frac{1-\exp \left(-\alpha_{\omega}\right)}{2} .
$$

Such a definition allows us to progressively and smoothly activate (and deactivate) assistance within the interval $\left[d_{\mathbf{A}_{\text {max }}}, d_{\mathbf{A}_{\text {min }}}\right]$ (see Fig. 7).

1) Compatibility with user intention estimation: We see that the motion planner will take control only if the wheelchair is at risk of collision with an obstacle or at risk of disturbance
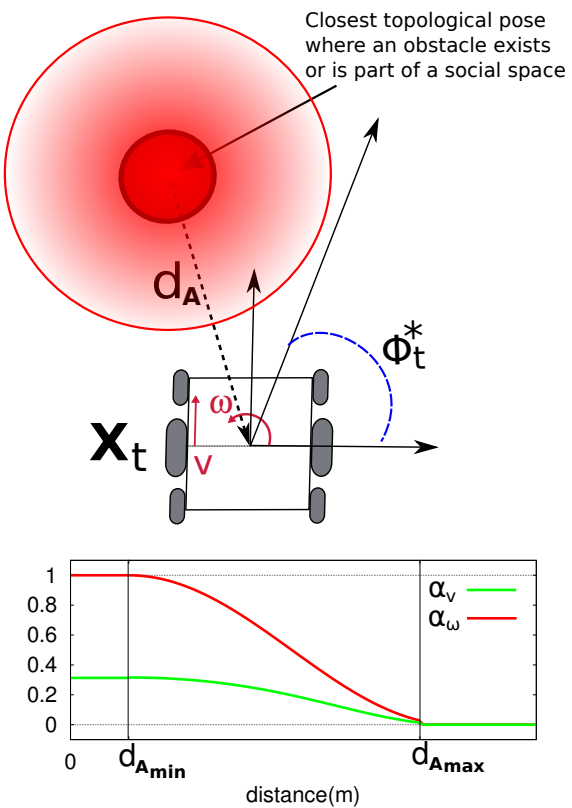

Fig. 7. The illustration on top provides an intuitive idea of the evolution of the factor $\alpha_{\omega}$ with respect to a detected obstacle (or social space). The graph at the bottom shows the related functions $\alpha_{v}$ and $\alpha_{\omega}$ with respect to the distance to the closest topological pose where an objects exists or which is part of a social space.

to others. Control is soon returned as danger is evaded. Moreover, we hypothesize that since the goal prediction window is short term and dependent on $\mathbf{X}_{t}$ and $\phi_{t}^{*}$, the specific case of $\mathbf{A} \neq \mathbf{0}_{2,2}$ at the goal prediction instant will not lead to erratic motions.

2) Stability: An analysis of stability of the formulation is essential in order to keep the system user centric and acceptable to patients. It was proved by Wang et.al. [28] that given that the set of feasible inputs from the user (i.e. $\left.\mathbf{V}_{o p}=\left\{\mathbf{v}_{o p}\right\}, \forall \mathbf{v}_{o p}\right)$ and the set of feasible robot motion commands (i.e. $\mathbf{V}_{r}=\left\{\mathbf{v}_{r}\right\}, \forall \mathbf{v}_{r}$ ) is convex, the controller $\mathbf{v}=\left(\mathbb{I}_{2}-\mathbf{A}\right) \mathbf{v}_{o p}+\mathbf{A} \mathbf{v}_{r}$ is stable in the sense of Lyapunov unless $\mathbf{V}_{o p} \cap \mathbf{V}_{r}=\emptyset$. If the motion planner generates motion commands $\left(\mathbf{v}_{r}\right)$ bounded by the maximum permissible velocities of the wheelchair, the instability condition is never encountered.

A simple approach taken by Dragan et al. [29] (within the context of manipulation) considering the same control sharing formulation, proved that an inescapable local minima does not occur unless $\forall \mathbf{v}_{o p}, \mathbf{v}_{r}=-k \mathbf{v}_{o p}$ for any $k \geq 0$ and $\alpha_{v}=\frac{1}{k+1}$ as well as $\alpha_{\omega}=\frac{1}{k+1}$. Within the proposed formulation, this case does not occur at all since at no point where $\alpha_{v}>0$ and $\alpha_{\omega}>0$ is $\alpha_{v}=\alpha_{\omega}$.

3) Full robot control is never taken: It can be seen from Eqn (9) that $\alpha_{v}$ never goes to its maximum value of 1 . We here hypothesize that altering the angular velocity $(\omega)$ of the wheelchair is sufficient for efficiently eliminating the risks of collision and disturbance. This also allows for a trajectory correction in the direction of the user intended motion as the user will have most of the control over the translational velocity. This also means that the user will be able to collide 
with an obstacle or move into the social spaces if he/she tries hard enough. This property is required firstly in order to keep the primary control with the user, secondly to learn the user habits and finally to teach the user how to efficiently operate a wheelchair [30].

4) Gateway to learning and teaching: An essential case to consider while designing mobility assistance systems is to facilitate the learning of user habits. For example, since the formulation (7) is directly dependent on the distance to the closest dangerous pose, the interval $\left[d_{\mathbf{A}_{\text {max }}}, d_{\mathbf{A}_{\text {min }}}\right]$ can be optimised online by reasoning within user teleoperation data and the assistance function $\mathbf{A}$. This could allow the motion controller to assist depending on the handicap and needs of the user. On the other hand, it is also advantageous to teach the users how to optimally use the system for better quality of experience [30]. As theorized by Dragan et. al. [29], we can provide the assistance $\mathbf{A}$ depending on the confidence in whether the user is intending to navigate to a specific goal $\mathbf{X}_{g}$. This confidence is of course obtained from the argument of Eqn. (5) where $\mathbf{X}_{i}=\mathbf{X}_{g}$. If $P_{g}$ represents this confidence, we can alter controller (7) as $\mathbf{v}=\left(\mathbb{I}_{2}-P_{g} \mathbf{A}\right) \mathbf{v}_{o p}+P_{g} \mathbf{A} \mathbf{v}_{r}$. In such a scenario, the system does not provide assistance unless it is confident of the user's intention thus inadvertently rewarding the user for good driving with smooth assistance.

\section{ANALYSIS}

\section{A. Simulations}

Our goal is to quantitatively analyse the proposed framework and also to try and verify the hypotheses put forward. An able bodied user rendered with a right weak signal ${ }^{1}$ teleoperates a robot in simulation using a 2D joystick (Logitech Gamepad F310). The user is tasked with following a corridor and then joining a human group in a hallway (see. Fig. 8). This task is particularly difficult for a person with a right weak signal as he has to turn towards the hallway while avoiding collision with the human as well as the wall.

The simulation was carried out within a ROS framework and the calculations were performed using the ViSP software [31]. In order to provide assistance based on the proposed framework, the parameters for searching the random goal were set as $r_{\min }=1.5 \mathrm{~m}, r_{\max }=3.5 \mathrm{~m}$ and $\phi_{r}=0.18 \mathrm{rad}$. The interval for considering a goal was set as $d_{\min }=0.5 \mathrm{~m}$ and $d_{\max }=3 \mathrm{~m} . \eta$ was set as 0.5 (see Eqn. (3)). Only a single goal pose $\left(\mathbf{X}_{1}\right)$ was pre-set for the simulation: the meeting point for the group where the robot should reach in order to equitably share space among the group [32]. The probability $P\left(\mathbf{X}_{1} \mid \mathbf{X}_{t}\right)$ was designed as $\max \left(0,1-\frac{d\left(\mathbf{X}_{t}, \mathbf{X}_{1}\right)}{3}\right)$. Therefore the pre-set goal is not considered unless $d\left(\mathbf{X}_{t}, \mathbf{X}_{1}\right) \leq 3 \mathrm{~m}$. Finally we set $d_{\mathbf{A}_{\text {max }}}=3 \mathrm{~m}$ and $d_{\mathbf{A}_{\text {min }}}=0.5 \mathrm{~m}$

Fig. 9 shows specific important frames for a single trial of the task. We see from Fig. 9(a), a random short term goal being predicted as the user follows the corridor, which is

\footnotetext{
${ }^{1}$ A right weak signal commonly occurs in people who have suffered stroke on their right side. In such case, the users are not able to give a strong signal on the right side and the user teleoperation is distorted as follows: $\omega_{o p}=\omega_{t h}$ if $\omega_{o p}<\omega_{t h}$ and $v_{o p}=v_{o p} \forall \mathbf{v}_{o p}=\left[v_{o p}, \omega_{o p}\right]^{T} . \omega_{t h}=-0.1 \mathrm{rad} / \mathrm{s}$ is a threshold value.
}

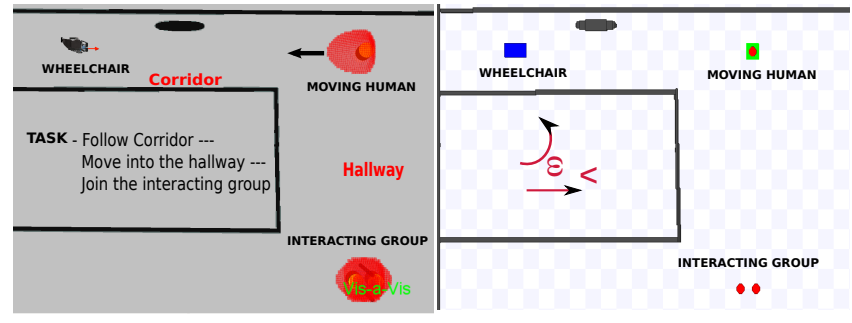

Fig. 8. The simulation scenario (Left) and the view of the user (Right). The user is not explicitly aware of the personal and interaction spaces around the humans.

TABLE I

COMPARISON OF QUANTITATIVE FACTORS (LOWER THE BETTER)

\begin{tabular}{c||c|c|c}
\hline Framework & AvgC & AvgS & AvgEx \\
\hline \hline No Assistance & 0.2 & 1.6 & 20.98 \\
Uniform control sharing & 0 & 0 & 16.56 \\
Proposed control sharing & $\mathbf{0}$ & $\mathbf{0}$ & $\mathbf{1 4 . 5 1}$
\end{tabular}

consistent with the user intended motion. Fig. 9(b) shows a frame where the user avoids the personal space of the human owing to the control sharing policy. In Fig. 9(c), we see a change in goal while assistance is still being provided (i.e $\left.\mathbf{A} \neq \mathbf{0}_{2,2}\right)$. But we observe from Figs. 10(c) and 10(d) that there is no erratic velocity changes due to the fact that the goals predicted are short term and compliant with the user intended direction. This is consistent with our hypothesis stated in Sec. VB, Part 1. Finally Fig. 9(d) shows the pre-set goal being predicted as the user moves onto join the group.

We also test three control (sharing) frameworks: No assistance, Uniform assistance (i.e. $\alpha_{v}=\alpha_{\omega}$ ), and the proposed framework (Eqn. (7)). The user performs 5 trials for each framework. Three factors are selected for analysis: average number of collisions with obstacles (AvgC), average number of encroachment of personal/interaction space (AvgS) and the average user exertion (AvgEx). The user exertion for each trial was calculated as $\sum_{t}\left|\omega_{o p}-\omega_{t h}\right|, \forall \omega_{o p}<\omega_{t h}{ }^{2}$. The results are tabulated in Table I.

We can see that the shared control approaches are successful in avoiding collisions as well as avoiding social space encroachment. Moreover, our proposed approach performs equally as the uniform control sharing framework in terms of avoiding collision and encroachment thus verifying our hypothesis that a correction in angular velocity is enough in order to avoid danger. But user exertion is reduced while using the proposed control sharing framework. This is in part due to the fact that the correction is in the direction of the user intention motion (see Figs. 10(c) and 10(d)). From the angular velocity plots (Figs. 10(b) and 10(d)), we can also asses the level of exertion in terms of the user trying to signal $\omega_{o p}<\omega_{t h}$. Importantly, the evolution of the factors $\alpha_{v}$ and $\alpha_{\omega}$ (Figs. 10(c) and 10(d)) show a progressive increase (and decrease) which is essential for better quality of experience.

\footnotetext{
${ }^{2}$ It can be intuitively seen as the stress of the user trying to signal an angular velocity of $\omega_{o p}<\omega_{t h}$.
} 


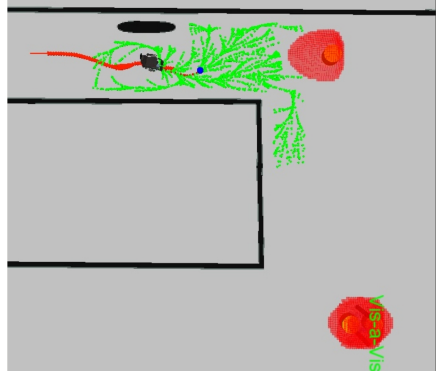

(a) Predicting a random goal

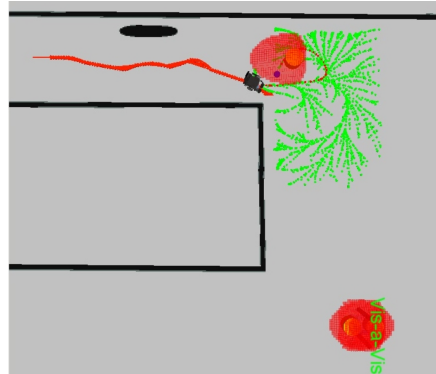

(b) Avoiding the personal space

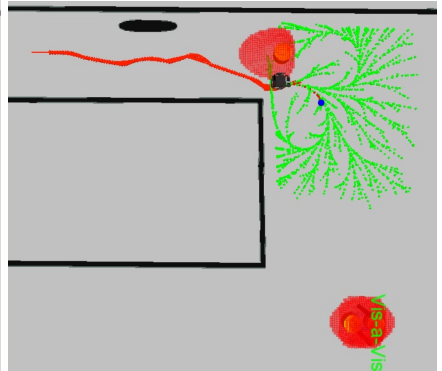

(c) Change in goal while $\mathbf{A} \neq \mathbf{0}_{2,2}$

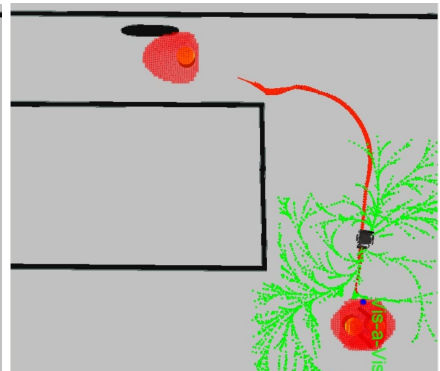

(d) Prediction of pre-set goal

Fig. 9. Important frames captured during the task execution using the semi-autonomous framework.

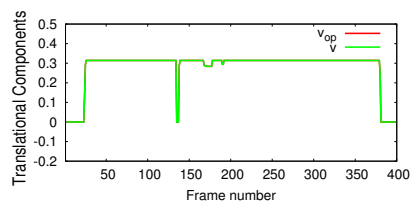

(a) $v_{o p}$ and $v$ - No assistance

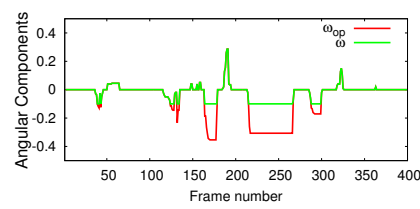

(b) $\omega_{o p}$ and $\omega-$ No assistance

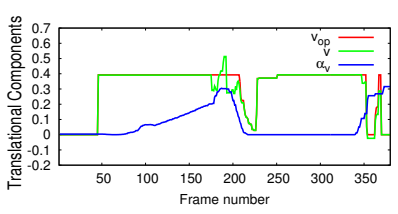

(c) $v_{o p}, v$ and $\alpha_{v}$ - With assistance

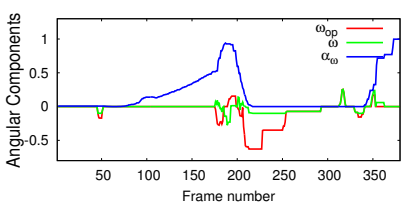

(d) $\omega_{o p}, \omega$ and $\alpha_{\omega}-$ With assistance

Fig. 10. The evolution of the velocity components during the task execution.

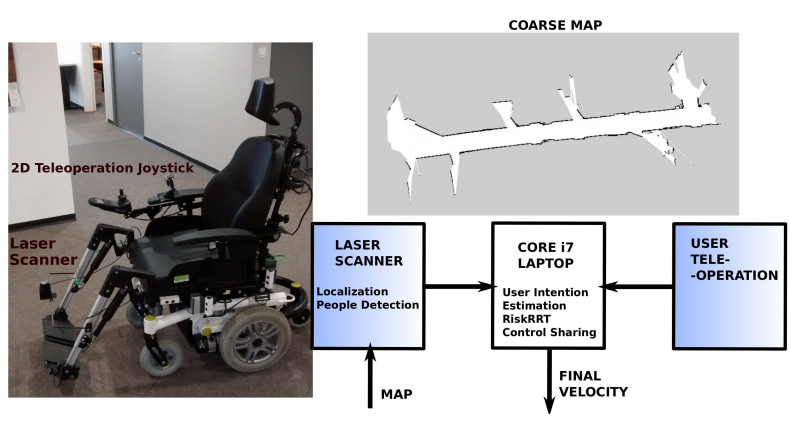

Fig. 11. Experimental setup along with the coarse map of the corridor created using the laser.

\section{B. Experiments}

The framework was tested on an off-the-shelf powered wheelchair (You-Q Luca) which could be teleoperated using a standard joystick coming from Penny \& Giles. A Hokuyo URG-04LX Laser Scanner was equipped as shown Fig. 11. As the only exteroceptive sensor, the laser scanner was used to localize the wheelchair and also to detect people. Initially a coarse map of the operating environment (a long corridor) was created (Fig. 11). Since we don't use odometry, a scan matching technique [33] was used to localize within the map. Such a map is not required if odometry can be leveraged alongside a Simultaneous Localization and Mapping (SLAM) scheme. A laser-based leg tracking algorithm was used to classify and track people. The objective of conducting such an experiment is to try and prove the efficacy of the proposed framework in an off-the-shelf real world system.

Again the framework was designed within ROS and the calculations were performed on an off-board core i7 laptop (connected via ethernet) using ViSP. Such a setup amounted to a $15 \mathrm{~ms}$ latency from user teleoperation to the motion

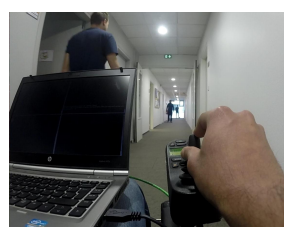

(a) Human 1

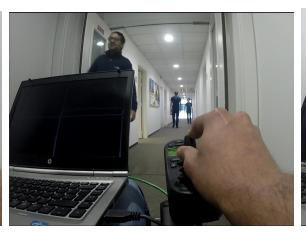

(b) Human 2

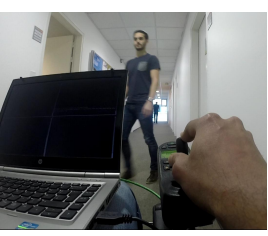

(c) Human 3 - Moving away from his own path
Fig. 12. Frames captured during one of the tests that depict encounters of the wheelchair with humans while following a corridor.
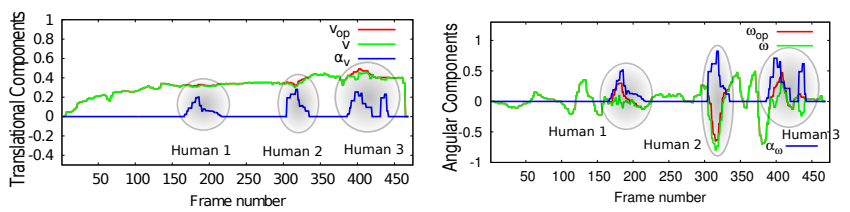

Fig. 13. The evolution of the velocity components during the experiment execution. We can observe the progressive increase of the factors $\alpha_{v}$ and $\alpha_{\omega}$.

controller which is undetectable to the users. The random goal and the goal consideration window parameters were kept the same as in the simulations. But no goal was pre-set in the map. Therefore all estimated goals were random short term goals.

An able bodied user with a simulated right weak signal (with $\omega_{t h}=-0.7 \mathrm{rad} / \mathrm{s}$, see 1) was tasked with following along the corridor in the presence of moving humans. Fig. 12 shows instances from one execution of the task when the wheelchair is in operating close to humans. These instances are highlighted in the velocity plots (Fig. 13) where we can see that the user teleoperation is augmented as expected in order to avoid the personal space of the moving humans in order to reduce the risk of disturbance. 
Discussion: We observed that while testing with human volunteers, in most cases, they were polite enough to move out of the way of the wheelchair (see Fig 12(c)). Since human perception of the wheelchair behaviour is not considered within the framework, it would be also advantageous to subjectively analyse this in order to create a more optimal solution.

\section{CONCLUSiOn AND FUture WORK}

We presented an adaptive semi-autonomous framework for wheelchair navigation assistance in human environments. A socially compliant motion planner was leveraged to augment the user teleoperation so that progressive assistance could be provided for safe navigation. Generalized formulations for estimating short term user intentions and for sharing control were provided. The system was quantitatively analysed in simulation wherein the effectiveness of the framework was proved. Experimental results corroborated the analyses and also showed the adaptability of the system in a real-world deployment. A public ROS package for RiskRRT has already been published. A new ROS package for the semiautonomous framework will be published soon. In the future, tests will be conducted with wheelchair users at a rehabilitation institute (Pole St. Helier, Rennes, France) able to operate the wheelchair while the laser scanner is mounted. Subjective measures regarding human perception and reaction to the behaviour of the wheelchair will also be analysed alongside the tests.

\section{ACKNOWLEDGMENT}

This work is supported by Inria Large-scale initiative action Personally Assisted Living (PAL). Authors would also like to thank François Pasteau who is with Ergovie, France.

\section{REFERENCES}

[1] M. W. Brault, "Americans with disability 2010," United States Census Bureau, 2012.

[2] T. Gomi and A. Griffith, "Developing intelligent wheelchairs for the handicapped," Assistive Technology and Artificial Intelligence, Springer Berlin Heidelberg, 1998, vol. 1458, pp. 150-178.

[3] S. P. Levine, D. A. Bell, L. A. Jaros, R. C. Simpson, Y. Koren, and J. Borenstein, "The NavChair Assistive Wheelchair Navigation System," IEEE Trans. on Rehabilitation Engineering, vol. 7, pp. 443451, 1999.

[4] E. Demeester, E. EB Vander Poorten, A. Hüntemann, and J. De Schutter, "Wheelchair navigation assistance in the fp7 project radhar: Objectives and current state," IROS Workshop on Progress, Challenges and Future Perspectives in Navigation and Manipulation Assistance for Robotic Wheelchairs, 2012.

[5] A. Kokosy, T. Floquet, G. Howells, H. Hu, M. Pepper, and C. Donzé, "SYSIASS An Intelligent Powered Wheelchair," Int. Conf. on Systems and Computer Science, 2012.

[6] B. D. Argall, “ Modular and Adaptive Wheelchair Automation." The 14th Int. Symposium on Experimental Robotics, 2016.

[7] V. Narayanan, F. Pasteau, M. Marchal, A. Krupa, and M. Babel, "Vision-based adaptive assistance and haptic guidance for safe wheelchair corridor following," Computer Vision and Image Understanding, To appear, 2016.

[8] J. Rios-Martinez, A. Spalanzani, and C. Laugier, "Understanding human interaction for probabilistic autonomous navigation using Risk-RRT approach," IEEE/RSJ Int. Conf. on Intelligent Robots and Systems, 2011, pp. 2014-2019.

[9] A. Escobedo, A. Spalanzani, and C. Laugier, "Using social cues to estimate possible destinations when driving a robotic wheelchair," EEE/RSJ Int. Conf. on Intelligent Robots and Systems , 2014, pp. 3299-3304.
[10] D. Vasquez, P. Stein, J. Rios-Martinez, A. Escobedo, A. Spalanzani, and C. Laugier, "Human Aware Navigation for Assistive Robotics," Int. Symposium on Experimental Robotics vol. 88, 2013.

[11] T. Carlson and J. Del R Millan, "Brain-controlled wheelchairs: A robotic architecture," IEEE Robotics Automation Magazine, vol. 20, no. 1, pp. 65-73, 2013.

[12] B. Kim and J. Pineau, "Socially adaptive path planning in human environments using inverse reinforcement learning," Int. Journal of Social Robotics, vol. 8, no. 1, pp. 51-66, 2015.

[13] L. Nordenfelt, Dignity in Care for Older People, Wiley-Blackwell, 2009.

[14] E. T. Hall. The hidden dimension, Doubleday, 1966.

[15] T. Taha, J. Miro, and G. Dissanayake, "POMDP-based long-term user intention prediction for wheelchair navigation," IEEE Int. Conf. on Robotics and Automation, pp. 3920-3925, 2008.

[16] A. Goil, M. Derry, and B. Argall, "Using machine learning to blend human and robot controls for assisted wheelchair navigation," IEEE Int. Conf. on Rehabilitation Robotics, pp. 1-6, 2013

[17] D. Karreman, L. Utama, M. Joosse, M. Lohse, B. van Dijk, and V. Evers, "Robot etiquette: How to approach a pair of people?" ACM/IEEE Int. Conf. on Human-robot Interaction, pp. 196-197, 2014.

[18] P. Althaus, H. Ishiguro, T. Kanda, T. Miyashita, and H. Christensen, "Navigation for human-robot interaction tasks," IEEE Int. Conf. on Robotics and Automation, 2004.

[19] T. Kruse, A. K. Pandey, R. Alami, and A. Kirsch, "Human-aware robot navigation: A survey," Robotics and Autonomous Systems, vol. 61, no. 12, pp. 1726-1743, 2013.

[20] J. Rios-Martinez, A. Spalanzani, and C. Laugier, "From Proxemics Theory to Socially-Aware Navigation: A Survey," Int. Journal of Social Robotics, 2014.

[21] M. Walters, K. Dautenhahn, S. Woods, and K. Koay, "Robotic etiquette: Results from user studies involving a fetch and carry task," $n d$ ACM/IEEE Int. Conf. on Human-Robot Interaction, pp. 317-324, 2007.

[22] I. Nishitani, T. Matsumura, M. Ozawa, A. Yorozu, and M. Takahashi, "Human-centered space path planning for mobile robot in dynamic environments," Robotics and Autonomous Systems, vol. 66, pp. 18 26, 2015.

[23] T. Carlson and Y. Demiris, "Collaborative control for a robotic wheelchair: Evaluation of performance, attention, and workload," IEEE Trans. on Systems, Man, and Cybernetics, Part B: Cybernetics, vol. 42, no. 3, pp. 876-888, 2012.

[24] E. Demeester, A. Huntemann, D. Vanhooydonck, G. Vanacker, A. Degeest, H. Van Brussel, and M. Nuttin, "Bayesian estimation of wheelchair driver intents: Modeling intents as geometric paths tracked by the driver," IEEE/RSJ Int. Conf. on Intelligent Robots and Systems, pp. 5775-5780, 2006.

[25] C. Fulgenzi, A. Spalanzani, C. Laugier, and C. Tay, "Risk based motion planning and navigation in uncertain dynamic environment, Research Report, 2010.

[26] Y. Kanayama, Y. Kimura, F. Miyazaki, and T. Noguchi, "A stable tracking control method for a non-holonomic mobile robot," IEEE/RSJ Int. Conf. on Intelligent Robots and Systems, pp. 1236-1241, 1991.

[27] B. D. Ziebart, A. Maas, J. A. Bagnell, and A. K. Dey, "Maximum entropy inverse reinforcement learning," in Proc. of AAAI, pp. 1433$1438,2008$.

[28] H. Wang and X. Liu, "Adaptive shared control for a novel mobile assistive robot," IEEE/ASME Trans. on Mechatronics, vol. 19, no. 6, pp. 1725-1736, 2014.

[29] A. Dragan and S. Srinivasa, "Formalizing assistive teleoperation," Robotics: Science and Systems, 2012.

[30] L. Montesano, J. Minguez, J. M. Alcubierre, and L. Montano, "Towards the adaptation of a robotic wheelchair for cognitive disabled children," IEEE/RSJ Int. Conf. on Intelligent Robots and Systems, pp. 710-716, 2006.

[31] E. Marchand, F. Spindler, and F. Chaumette, "ViSP for visual servoing: a generic software platform with a wide class of robot control skills," IEEE Robotics and Automation Magazine, vol. 12, no. 4, pp. 40-52, 2005.

[32] V. K. Narayanan, A. Spalanzani, F. Pasteau, and M. Babel, "On equitably approaching and joining a group of interacting humans," in IEEE/RSJ Int. Conf. on Intelligent Robots and Systems, pp. 4071-4077, 2015.

[33] A. Censi, "An ICP variant using a point-to-line metric," in IEEE Int. Conf. on Robotics and Automation, 2008. 